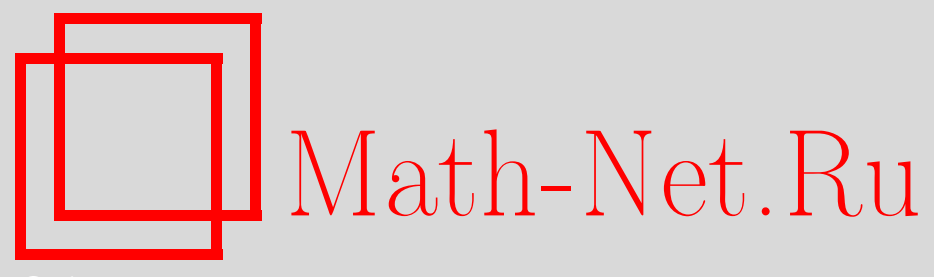

Н. В. Лазакович, О. Л. Яблонский, Предельное поведение итовских конечных сумм с осреднением, Теория вероятн. и ее примен., 2005, том 50, выпуск 4, 711-732

DOI: https://doi.org/10.4213/tvp126

Использование Общероссийского математического портала MathNet.Ru подразумевает, что вы прочитали и согласны с пользовательским соглашением

http://www . mathnet.ru/rus/agreement

Параметры загрузки:

IP: 107.22 .136 .117

26 апреля 2023 г., 04:02:37

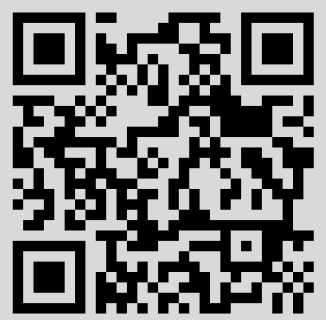




\section{ПРЕДЕЛЬНОЕ ПОВЕДЕНИЕ ИТОВСКИХ КОНЕЧНЫХ СУММ С ОСРЕДНЕНИЕМ ${ }^{1)}$}

В работе рассматривается предельное поведение сумм с осреднением, содержащих стохастические процессы Леви. Для этого вводится класс стохастических интегралов для процессов Леви, содержащий, в частности, стохастические интегралы Ито, Стратоновича и др. С помощью введенных интегралов дана полная классификация предельного поведения рассматриваемых сумм.

Ключевые слова и фразы: алгебра обобщенных случайных процессов, процесс Леви, стохастический интеграл.

Введение. Для исследования решений дифференциальных уравнений, содержащих обобщенные случайные процессы типа «белого шума», разработана специальная теория стохастических дифференциальных уравнений. Данная теория базируется на понятиях стохастических интегралов Ито [1] и Стратоновича (последний называют еще симметрическим стохастическим интегралом или интегралом Стратоновича-Фиска) [2], [3], а также на их уточнениях и обобщениях - стохастическом интеграле Огавы [4], стохастическом $\theta$-интеграле [5, гл. $3, \S 4]$ и др.

Вместе с тем имеется много работ, авторы которых исследуют решения стохастических дифференциальных уравнений обычными с позиции неслучайного анализа методами на аппроксимационном уровне. При этом, как правило, решения уравнений Ито приближаются решениями соответствующих конечно-разностных уравнений $[6$, гл. $5, \S 4]$, а решения уравнений Стратоновича - решениями обыкновенных дифференциальных уравнений $[7],[8$, гл. $6, \S 7]$. $\mathrm{K}$ исследованиям подобного типа необходимо отнести и работы, в которых авторы приближают стохастические интегралы «оригинальными» суммами (см., например, монографию $[9$, гл. $5, \S 5]$ и цитируемую в ней литературу). При переходе к уравнениям в этом случае приходится иметь дело с конечноразностными уравнениями в неявной форме и для изучения решений прибегать к осреднению.

* Белорусский государственный университет, механико-математический факультет, пр. Фр. Скорины, 4, 220050 Минск, Беларусь; e-mail: lazakovich@bsu.by; yablonski@bsu.by

1) Работа выполнена при поддержке INTAS (грант № 03-55-1861). 
Следует отметить, что проблемы теории стохастических дифференциальных уравнений непосредственно связаны с проблемой умножения обобщенных функций. Такие уравнения содержат произведение обобщенных функций на недостаточно гладкие функции. В сообщении [10] анонсирована конструкция алгебры обобщенных случайных процессов, позволяющая с единых позиций изучать решения различных классов стохастических дифференциальных уравнений, а в статье [11] показано, что как решения стохастических уравнений Ито, так и решения уравнений Стратоновича могут быть аппроксимированы решениями соответствующего уравнения в дифференциалах в этой алгебре. При этом исследование ассоциированных решений уравнений в дифференциалах сводится к описанию предельного поведения итовских конечных сумм с осреднением следующего вида:

$$
\sum_{k=0}^{m-1} f_{n}\left(L_{n}\left(t_{k}\right)\right)\left[L_{n}\left(t_{k+1}\right)-L_{n}\left(t_{k}\right)\right]
$$

где $\left\{t_{0}, t_{1}, \ldots, t_{m}\right\}$ - разбиение отрезка $[0 ; t], t_{k+1}-t_{k}=h_{n}, k=$ $0,1, \ldots, m-1, t \in \mathbf{T}=[0 ; T], T \in \mathbf{R}$, а $f_{n}$ и $L_{n}-$ свертка функции $f$ и случайного процесса $L$ соответственно с «шапочкой» $[12$, гл. $2, \S 5]$, т.е., например, для $L_{n}$,

$$
L_{n}(t)=\left(L * \rho_{n}\right)(t)=\int_{0}^{1 / n} L(t+s) \rho_{n}(s) d s, \quad t \in \mathbf{T},
$$

где $\rho_{n}(t) \in \mathbf{C}^{\infty}(\mathbf{R}), \rho_{n}(t) \geqslant 0, \operatorname{supp} \rho_{n}(t) \subset[0 ; 1 / n], \int_{0}^{1 / n} \rho_{n}(s) d s=1$.

В статьях [13]-[16] показано, что предельное поведение сумм (1) в случае, когда $L(t)$ - стандартный процесс броуновского движения $[8$, гл. $1, \S 7]$, полностью описывается стохастическими $\theta$-интегралами $[5$, гл. $3, \S 4]$ вида

$$
\int_{0}^{t} f(L(s)) d L(s), \quad t \in \mathbf{T}
$$

в зависимости от связи между $h_{n}$ и $1 / n$.

В сообщении [17] данная задача рассматривалась в случае, когда $L(t)$ - пуассоновский случайный процесс $[8$, гл. $1, \S 9]$, а «шапочки» $\rho_{n}, n \geqslant 1$, имеют стандартный вид, т.е. $\rho_{n}(t)=n \rho(n t), t \in[0 ; 1]$. В этом случае предельное поведение сумм (1) описывается либо интегралом Ито вида $(2)$ - если $1 / n=o\left(h_{n}\right)$ при $n \rightarrow \infty, h_{n} \rightarrow 0$, либо новым стохастическим интегралом, который автор назвал симметрическим, если $h_{n}=o(1 / n)$.

Настоящая работа посвящена исследованию предельного поведения сумм вида $(1)$, если $L(t)$ - квадратично интегрируемый случайный процесс Леви. Запаса существующих стохастических интегралов для описания пределов данных сумм оказалось недостаточно. В статье вводится новый класс стохастических интегралов - стохастические $\mu$-интегралы, 
которые являются обобщением и уточнением (в разрывном случае) стохастических интегралов Ито, Стратоновича, $\theta$-интегралов, и в терминах этих интегралов дается полное описание предельного поведения итовских конечных сумм с осреднением (1).

1. Основные результаты. Пусть $(\Omega, \mathscr{A}, \mathbf{P})$ - полное вероятностное пространство, $L(t)=\left(L_{1}(t) ; L_{2}(t)\right), t \in \mathbf{T}=[0, T], T \in \mathbf{R}$, двумерный случайный процесс Леви, т.е. выходящий из нуля непрерывный справа и имеющий конечные пределы слева процесс с независимыми стационарными приращениями. Предположим, что поток $\sigma$ алгебр $\left\{\mathscr{F}_{t}\right\}_{t \in \mathbf{T}}$ порожден траекториями $L(t), t \in \mathbf{T}$. По теореме Леви (см., например, [8, гл. $2, \S 4])$ существуют $d$-мерное $\mathscr{F}_{t}$-броуновское движение $B(t)=\left(B_{k}(t)\right)_{k=1}^{d}, 0 \leqslant d \leqslant 2,(2 \times d)$-матрица $A=\left(a_{i k}\right)$ ранга $d$, двумерный вектор $\mathbf{b}=\left(b_{1} ; b_{2}\right)$ и стационарная $\mathscr{F}_{t}$-пуассоновская мера $N(d s d x)$, для которых $L(t)$ представим в виде

$$
\begin{aligned}
L_{i}(t)= & \sum_{k=1}^{d} a_{i k} B_{k}(t)+b_{i} t+\int_{0}^{t+} \int_{\mathbf{R}^{2} \backslash\{0\}} x_{i} I_{(|x| \geqslant 1)} N(d s d x) \\
& +\int_{0}^{t+} \int_{\mathbf{R}^{2} \backslash\{0\}} x_{i} I_{(|x|<1)} \tilde{N}(d s d x), \quad i=1,2 .
\end{aligned}
$$

Здесь $x=\left(x_{1} ; x_{2}\right) \in \mathbf{R}^{2}$ и $\tilde{N}(d s d x)=N(d s d x)-\widehat{N}(d s d x)$ - компенсированная пуассоновская мера, компенсатор $\widehat{N}(d s d x)$ которой имеет вид $\widehat{N}(d s d x)=\mathbf{E} N(d s d x)=d s n(d x)$ и $\sigma$-конечная мера $n(d x)$ обладает следуюшим свойством:

$$
\int_{\mathbf{R}^{2} \backslash\{0\}} \frac{|x|^{2}}{1+|x|^{2}} n(d x)<\infty .
$$

О п р е д е л ен и е 1. Пусть $f(x) \in \mathbf{C}^{2}(\mathbf{R})$ и $\mu$ - произвольная вероятностная мера на отрезке $[0 ; 1]$. Стохастическим $\mu$-интегралом назовем случайный процесс

$$
\begin{array}{rl}
(\mu) \int_{0}^{t+} f\left(L_{1}(s-)\right) d L_{2}(s)=\lim _{|\sigma| \rightarrow 0} \sum_{k=0}^{m-1} \int_{0}^{1} & f\left(L_{1}\left(t_{k}\right)+u\left(L_{1}\left(t_{k+1}\right)-L_{1}\left(t_{k}\right)\right)\right) \\
& \times \mu(d u)\left(L_{2}\left(t_{k+1}\right)-L_{2}\left(t_{k}\right)\right),
\end{array}
$$

где $|\sigma|=\max _{0 \leqslant k \leqslant m-1}\left|t_{k+1}-t_{k}\right|, 0=t_{0}<t_{1}<\cdots<t_{m}=t-$ разбиение отрезка $[0 ; t], t \in \mathbf{T}$, и предел берется по вероятности.

3 а м е ч а н и е 1 . Определение $\mu$-интеграла можно распространить и на более широкие классы случайных процессов. Отметим, что предельное поведение конечных сумм из определения 1 в случае, когда $L_{1}$ - непрерывный, а $L_{2}$ - произвольный семимартингал, исследуется в $[9$, гл. $5, \S 5]$.

Данное определение корректно в силу следующего утверждения. 
Предложение 1. Пусть $f \in \mathbf{C}^{2}(\mathbf{R})$, погда

$$
\begin{aligned}
& (\mu) \int_{0}^{t+} f\left(L_{1}(s-)\right) d L_{2}(s)=\sum_{k=1}^{d} a_{2 k} \int_{0}^{t} f\left(L_{1}(s)\right) d B_{k}(s) \\
& +b_{2} \int_{0}^{t} f\left(L_{1}(s)\right) d s+\theta \sum_{k=1}^{d} a_{1 k} a_{2 k} \int_{0}^{t} f^{\prime}\left(L_{1}(s)\right) d s \\
& +\int_{0}^{t+} \int_{\mathbf{R}^{2} \backslash\{0\}} \int_{0}^{1} x_{2} I_{(|x| \geqslant 1)} f\left(L_{1}(s-)+x_{1} u\right) \mu(d u) N(d s d x) \\
& +\int_{0}^{t+} \int_{\mathbf{R}^{2} \backslash\{0\}} \int_{0}^{1} x_{2} I_{(|x|<1)} f\left(L_{1}(s-)+x_{1} u\right) \mu(d u) \tilde{N}(d s d x) \\
& +\int_{0}^{t+} \int_{\mathbf{R}^{2} \backslash\{0\}} x_{2} I_{(|x|<1)} \\
& \quad \times\left(\int_{0}^{1} f\left(L_{1}(s)+x_{1} u\right) \mu(d u)-f\left(L_{1}(s)\right)\right) \widehat{N}(d s d x),
\end{aligned}
$$

где $\theta=\int_{0}^{1} u \mu(d u)$.

3 а м е ч а н и е 2. Соотношение (3) можно переписать следуюшим образом:

$$
\begin{aligned}
(\mu) & \int_{0}^{t} f\left(L_{1}(s-)\right) d L_{2}(s)=\int_{0}^{t} f\left(L_{1}(s-)\right) d L_{2}(s) \\
& +\theta \int_{0}^{t} f^{\prime}\left(L_{1}(s)\right) d\left[L_{1} ; L_{2}\right]^{c}(s) \\
& +\sum_{0<s \leqslant t}\left(\int_{0}^{1} f\left(L_{1}(s-)+u \Delta L_{1}(s)\right) \mu(d u) \Delta L_{2}(s)-f\left(L_{1}(s-)\right) \Delta L_{2}(s)\right),
\end{aligned}
$$

где $\left[L_{1} ; L_{2}\right]^{c}-$ непрерывная часть квадратичной ковариации $\left[L_{1} ; L_{2}\right]$, $\Delta L_{i}(s)=L_{i}(s)-L_{i}(s-), i=1,2$, и $\theta=\int_{0}^{1} u \mu(d u)$ (cp., например, с $[9$, гл. $5, \S 5])$.

В дальнейшем мы ограничимся рассмотрением одномерного случая (т.е. $\left.L_{1}(t)=L_{2}(t)=L(t)\right)$, причем будем предполагать, что поток $\left\{\mathscr{F}_{t}\right\}_{t \in \mathbf{T}}$ порожден процессом $L(t)$, где $L(t) \in \mathbf{L}^{2}(\Omega, \mathscr{A}, \mathbf{P})$ и, следовательно, может быть представлен в виде

$$
L(t)=L^{c}(t)+\int_{0}^{t+} \int_{\mathbf{R}} x \tilde{N}(d s d x), \quad t \in \mathbf{T},
$$

где $L^{c}(t)=a B(t)+b t, a, b \in \mathbf{R}, B(t)$ - одномерный стандартный процесс $\mathscr{F}_{t}$-броуновского движения, $\tilde{N}(d s d x)=N(d s d x)-\widehat{N}(d s d x), N(d s d x)-$ стационарная $\mathscr{F}_{t}$-пуассоновская мера, компенсатор которой $\widehat{N}(d s d x)=$ $\mathbf{E} N(d s d x)=d s n(d x)$ и $n(d x)-\sigma$-конечная мера такая, что

$$
\int_{\mathbf{R}}|x|^{2} n(d x)<\infty
$$


З а м е ч а н и е 3. Справедливо следуюшее равенство:

$$
\begin{aligned}
(\mu) & \int_{0}^{t+} f(L(s-)) d L(s)=(\theta) \int_{0}^{t} f(L(s)) d L^{c}(s) \\
& +\int_{0}^{t+} \int_{\mathbf{R}} \int_{0}^{1} x f(L(s-)+x u) \mu(d u) \tilde{N}(d s d x) \\
& +\int_{0}^{t+} \int_{\mathbf{R}}\left(\int_{0}^{1} x f(L(s)+x u) \mu(d u)-x f(L(s))\right) \widehat{N}(d s d x),
\end{aligned}
$$

где $\theta=\int_{0}^{1} u \mu(d u), t \in \mathbf{T}$, а первый интеграл в правой части равенства стохастический $\theta$-интеграл [5, гл. $3, \S 4]$.

Из соотношения (5) можно сделать вывод, что в непрерывном случае стохастический $\mu$-интеграл совпадает с $\theta$-интегралом, где $\theta=\int_{0}^{1} u \mu(d u)$.

3 а м е ч а н и е 4 . Пусть мера $\mu$ сосредоточена в одной точке в нуле. Тогда стохастический $\mu$-интеграл совпадает с интегралом Ито, т.е.

$$
(\mu) \int_{0}^{t+} f(L(s-)) d L(s)=\int_{0}^{t+} f(L(s-)) d L(s) .
$$

3 а м е ч а н и е 5 . Пусть $\mu$ - мера Лебега. Тогда из формулы (5) вытекает, что $\mu$-интеграл в данном случае будет иметь вид

$$
\begin{aligned}
(\mu) & \int_{0}^{t+} f(L(s-)) d L(s)=\int_{0}^{t} f(L(s)) \circ d L^{c}(s) \\
& +\int_{0}^{t+} \int_{\mathbf{R}} \int_{0}^{1} x f(L(s-)+x u) d u \tilde{N}(d s d x) \\
& +\int_{0}^{t+} \int_{\mathbf{R}}\left(\int_{0}^{1} x f(L(s)+x u) d u-x f(L(s))\right) \widehat{N}(d s d x), \quad t \in \mathbf{T},
\end{aligned}
$$

где первый стохастический интеграл в правой части - стохастический интеграл Стратоновича. Заметим, что этот интеграл для специального класса процессов рассматривался в работах [18] и [19].

Для $\mu$-интеграла в равенстве (6) формула Ито принимает такой же вид, как и в обычном анализе, т.е.

$$
(\mu) \int_{0}^{t+} f^{\prime}(L(s-)) d L(s)=f(L(t))-f(L(0)) .
$$

Поэтому естественно назвать стохастический $\mu$-интеграл при $\mu(d u)=d u$ симметрическим интегралом или интегралом Стратоновича. В дальнейшем для такого интеграла используем обозначение $\int_{0}^{t+} f(L(s-)) \circ d L(s)$.

Известны следующие определения симметрического интеграла для скачкообразных случайных процессов - он либо полагается равным интегралу Ито $[9$, гл. $5, \S 4]$, либо определяется с помощью таких же интегральных сумм, как и для непрерывных случайных процессов $[5$, гл. $3, \S 4]$. Еше одна конструкция симметрического интеграла для специального класса процессов дана в работах [18] и [19]. На наш взгляд, 
определение (6) является более предпочтительным (см., например, пример из монографии $[20$, гл. $1, \S 3])$ и обобшает соответствующий интеграл из [18] и [19]. Следует отметить, что и упомянутые выше симметрические интегралы могут быть аппроксимированы суммами вида (1).

Аппроксимацию случайного процесса $L(t)$ возьмем в виде свертки

$$
L_{n}(t)=\left(L * \rho_{n}\right)(t)=\int_{0}^{1 / n} L(t+s) \rho_{n}(s) d s, \quad t \in \mathbf{T},
$$

где $\rho_{n}(t) \in \mathbf{C}^{\infty}(\mathbf{R}), \rho_{n}(t) \geqslant 0, \operatorname{supp} \rho_{n}(t) \subset[0 ; 1 / n], \int_{0}^{1 / n} \rho_{n}(s) d s=1$.

Введем в рассмотрение функцию

$$
F_{n}(t)=\int_{t}^{1 / n} \rho_{n}(s) d s, \quad t \in \mathbf{R} .
$$

Несложно видеть, что $0 \leqslant F_{n}(t) \leqslant 1$, причем $F_{n}(t)=0$ при $t \geqslant 1 / n$ и $F_{n}(t)=1$ при $t \leqslant 0$.

Обратную к функции $F_{n}(t)$ определим следующим образом:

$$
F_{n}^{-1}(u)=\sup \left\{s: F_{n}(s)=u\right\}, \quad u \in[0 ; 1] .
$$

Заметим, что $F_{n}^{-1}(0)=+\infty$.

Учитывая формулу (7), получаем представление

$$
L_{n}(t)=\int_{0}^{(t+1 / n)+} F_{n}(s-t) d L(s) .
$$

Пусть $h_{n}>0$, тогда для любого $t \in \mathbf{T}$ найдутся такие $\tau_{t} \in\left[0 ; h_{n}\right)$ и $m_{t} \in \mathbf{N}$, что $t=\tau_{t}+m_{t} h_{n}$.

В данной работе исследуется предельное поведение конечных сумм $S_{n} f(t)=\sum_{k=0}^{m_{t}-1} f_{n}\left(L_{n}\left(\tau_{t}+k h_{n}\right)\right)\left(L_{n}\left(\tau_{t}+(k+1) h_{n}\right)-L_{n}\left(\tau_{t}+k h_{n}\right)\right), \quad t \in \mathbf{T}$, где $f_{n}=f * \rho_{n}, f \in \mathbf{C}_{B}^{2}(\mathbf{R})$, а $\mathbf{C}_{B}^{2}(\mathbf{R})$ - множество всех функций на $\mathbf{R}$, которые два раза непрерывно дифференцируемы и ограничены вместе со всеми своими частными производными.

Подобные суммы возникают при решении уравнений в дифференциалах в алгебре обобщенных случайных процессов [11].

Теорема 1. Пусть $n \rightarrow \infty, h_{n} \rightarrow 0$, причем $n^{2} h_{n} \rightarrow \infty$. Последовательность сумм $S_{n} f(t)$ сходится в $\mathbf{L}^{2}(\Omega, \mathscr{A}, \mathbf{P})$ и равномерно по $t \in \mathbf{T}$ для всех функиии $f \in \mathbf{C}_{B}^{2}(\mathbf{R})$ тогда и только тогда, когда для всех $\delta \in(0 ; 1)$ последовательность функиий $F_{n}\left(F_{n}^{-1}(u)-\delta h_{n}\right)$ сходится слабо для $u \in[0 ; 1]$ и предел не зависит от $\delta$.

Здесь и всюду в дальнейшем слабая сходимость последовательности монотонных функций означает сходимость в каждой точке непрерывности предельной монотонной функции. 
О п р е д е л е н и е 2. Будем говорить, что функция $g$ принадлежит классу $\bar{G}(\underline{G})$, если существует последовательность попарно непересекающихся полуинтервалов $\left(a_{n} ; b_{n}\right] \subset[0 ; 1], n \in I$, где множество $I$ не более чем счетно, такая, что

$$
g(u)=\left\{\begin{array} { l l } 
{ b _ { n } , } & { u \in ( a _ { n } ; b _ { n } ] , } \\
{ u , } & { u \notin \bigcup _ { n \in I } ( a _ { n } ; b _ { n } ] }
\end{array} \quad \left(g(u)= \begin{cases}a_{n}, & u \in\left[a_{n} ; b_{n}\right), \\
u, & \left.u \notin \bigcup_{n \in I}\left[a_{n} ; b_{n}\right)\right) .\end{cases}\right.\right.
$$

Теорема 2. Пусть для всех $\delta \in(0 ; 1)$ последовательность функиий $F_{n}\left(F_{n}^{-1}(u)-\delta h_{n}\right)$ сходится слабо для $u \in[0 ; 1]$ nрu $n \rightarrow \infty, h_{n} \rightarrow 0$ $u n^{2} h_{n} \rightarrow \infty$ иредел не зависит от $\delta$, m.е. $F_{n}\left(F_{n}^{-1}(u)-\delta h_{n}\right) \rightarrow g(u)$, тогда функиия $g$ принадлежит классу $\bar{G} u$

$$
S_{n} f(t) \longrightarrow(\mu) \int_{0}^{t+} f(L(s-)) d L(s)
$$

в $\mathbf{L}^{2}(\Omega, \mathscr{A}, \mathbf{P})$ и равномерно по $t \in \mathbf{T}$ для всех функиий $f \in \mathbf{C}_{B}^{2}(\mathbf{R})$ nри $n \rightarrow \infty, h_{n} \rightarrow 0$ u $n^{2} h_{n} \rightarrow \infty$, где мера $\mu$ порождена функиией $g$.

Следствие 1. Пусть последовательность «шапочек» $\rho_{n}$ имеет вид $\rho_{n}(t)=n \rho(n t)$, где $\rho(t) \geqslant 0, \operatorname{supp} \rho(t) \subset[0 ; 1], \int_{0}^{1} \rho(s) d s=1$. Последовательность сумм $S_{n} f(t)$ сходится в $\mathbf{L}^{2}(\Omega, \mathscr{A}, \mathbf{P})$ и равномерно nо $t \in \mathbf{T}$ для всех функиий $f \in \mathbf{C}_{B}^{2}(\mathbf{R})$ nри $n \rightarrow \infty, h_{n} \rightarrow 0$ тогда $u$ только тогда, когда $1 / n=o\left(h_{n}\right)$ либо $h_{n}=o(1 / n)$. При этом

$$
\begin{aligned}
& S_{n} f(t) \longrightarrow \int_{0}^{t+} f(L(s-)) d L(s) \Longleftrightarrow \frac{1}{n}=o\left(h_{n}\right), \\
& S_{n} f(t) \longrightarrow \int_{0}^{t+} f(L(s-)) \circ d L(s) \Longleftrightarrow h_{n}=o\left(\frac{1}{n}\right) .
\end{aligned}
$$

Введем следующее обозначение:

$$
K\left(n ; h_{n}\right)=\iint_{\substack{0 \leqslant s, \tau \leqslant 1 / n \\|s-\tau| \leqslant h_{n}}}\left(1-\frac{|s-\tau|}{h_{n}}\right) \rho_{n}(s) \rho_{n}(\tau) d s d \tau .
$$

Непосредственно из определения функции $\rho_{n}(s)$ нетрудно заметить, что $0 \leqslant K\left(n ; h_{n}\right) \leqslant 1$.

Следствие 2. Пусть прочесс Леви $L(t)$ непрерьвен и функиия $f \in \mathbf{C}_{B}^{2}(\mathbf{R})$. Последовательность сумм $S_{n} f(t)$ сходится в $\mathbf{L}^{2}(\Omega, \mathscr{A}, \mathbf{P})$ u равномерно по $t \in \mathrm{T}$ при $n \rightarrow \infty, h_{n} \rightarrow 0$ тогда и только тогда, когда числовая последовательность $K\left(n ; h_{n}\right)$ из (10) сходится при $n \rightarrow \infty$, $h_{n} \rightarrow 0$. При этом

$$
S_{n} f(t) \rightarrow(\theta) \int_{0}^{t} f(L(s)) d L(s)
$$

если и только если $K\left(n ; h_{n}\right) \rightarrow(1-2 \theta)$, где $\theta \in\left[0 ; \frac{1}{2}\right]$. 
По аналогии с суммами $S_{n} f(t)$ рассмотрим суммы с опережением

$$
\begin{aligned}
S_{n}^{+} f(t)=\sum_{k=0}^{m_{t}-1} & f_{n}\left(L_{n}\left(\tau_{t}+(k+1) h_{n}\right)\right) \\
& \times\left(L_{n}\left(\tau_{t}+(k+1) h_{n}\right)-L_{n}\left(\tau_{t}+k h_{n}\right)\right), \quad t \in \mathbf{T},
\end{aligned}
$$

где, как и раньше, $f_{n}=f * \rho_{n}$ и $f \in \mathbf{C}_{B}^{2}(\mathbf{R})$.

Для непрерывных процессов такие суммы исследовались в рабо$\operatorname{tax}[14],[16]$. Для процесса $L(t)$ из (4) предельное поведение сумм $S_{n}^{+} f(t)$ описывается следующими теоремами.

Теорема 3. Последовательность сумм $S_{n}^{+} f(t)$ сходится в пространстве $\mathbf{L}^{2}(\Omega, \mathscr{A}, \mathbf{P})$ и равномерно по $t \in \mathbf{T}$ для всех функций $f \in \mathbf{C}_{B}^{2}(\mathbf{R}) n p u n \rightarrow \infty, h_{n} \rightarrow 0 u n^{2} h_{n} \rightarrow \infty$ mогда u только тогда, когда для всех $\delta \in(0 ; 1)$ последовательность Функиий $F_{n}\left(F_{n}^{-1}(u)+\delta h_{n}\right)$ сходится слабо для $u \in[0 ; 1]$ nрu $n \rightarrow \infty, h_{n} \rightarrow 0 u n^{2} h_{n} \rightarrow \infty$ иредел не зависит от $\delta$.

Теорема 4. Пусть для всех $\delta \in(0 ; 1)$ последовательность функиий $F_{n}\left(F_{n}^{-1}(u)+\delta h_{n}\right)$ сходится слабо для $u \in[0 ; 1]$ nри $n \rightarrow \infty, h_{n} \rightarrow 0$ $u n^{2} h_{n} \rightarrow \infty$ иредел не зависит от $\delta$, m.е. $F_{n}\left(F_{n}^{-1}(u)+\delta h_{n}\right) \rightarrow g(u)$, тогда функиия $g$ принадлежит классу $\underline{G} u$

$$
S_{n}^{+} f(t) \longrightarrow(\mu) \int_{0}^{t+} f(L(s-)) d L(s)
$$

в $\mathbf{L}^{2}(\Omega, \mathscr{A}, \mathbf{P})$ и равномерно по $t \in \mathbf{T}$ для всех функций $f \in \mathbf{C}_{B}^{2}(\mathbf{R}) n p u$ $n \rightarrow \infty, \quad h_{n} \rightarrow 0$ u $n^{2} h_{n} \rightarrow \infty$, где мера $\mu$ порождена функиией $g$.

Следствие 3. Пусть последовательность $\rho_{n}$ такая же, как и в следствии 1. Тогда для сходимости последовательности сумк $S_{n}^{+} f(t)$ в $\mathbf{L}^{2}(\Omega, \mathscr{A}, \mathbf{P})$ и равномерно по $t \in \mathbf{T}$ для всех функций $f \in \mathbf{C}_{B}^{2}(\mathbf{R})$ при $n \rightarrow \infty, h_{n} \rightarrow 0$ необходимо и достаточно, чтобь $1 / n=o\left(h_{n}\right)$ либо $h_{n}=o(1 / n)$. При этом

$$
\begin{aligned}
h_{n}=o\left(\frac{1}{n}\right) \Longleftrightarrow S_{n}^{+} f(t) \longrightarrow \int_{0}^{t+} f(L(s-)) \circ d L(s), \\
\frac{1}{n}=o\left(h_{n}\right) \Longleftrightarrow S_{n}^{+} f(t) \longrightarrow(\mu) \int_{0}^{t+} f(L(s-)) d L(s),
\end{aligned}
$$

где мера $\mu$ сосредоточена в точке 1.

Следствие 4. Пусть прочесс Леви $L(t)$ непрерывен и функиия $f \in \mathbf{C}_{B}^{2}(\mathbf{R})$. Последовательность сумм $S_{n}^{+} f(t)$ сходится в $\mathbf{L}^{2}(\Omega, \mathscr{A}, \mathbf{P})$ u равномерно по $t \in \mathbf{T}$ при $n \rightarrow \infty, h_{n} \rightarrow 0$ тогда и только тогда, когда числовая последовательность $K\left(n ; h_{n}\right)$ из (10) сходится $n р и n \rightarrow \infty$, $h_{n} \rightarrow 0$. При этом

$$
S_{n}^{+} f(t) \longrightarrow(\theta) \int_{0}^{t} f(L(s)) d L(s),
$$

если и только если $K\left(n ; h_{n}\right) \rightarrow(2 \theta-1)$; әде $\theta \in\left[\frac{1}{2} ; 1\right]$. 
Результаты следствий 2 и 4 впервые были доказаны в рабо$\operatorname{Tax}[14],[16]$.

3 а м е ч а н и е 6 . Отметим, что суммы $S_{n} f(t)$ и $S_{n}^{+} f(t)$ аппроксимируют только те $\mu$-интегралы, для которых мера $\mu$ порождена функциями из класса $G=\bar{G} \cup \underline{G}$. Для приближения остальных $\mu$-интегралов необходимо рассматривать более широкие классы аппроксимаций.

2. Вспомогательные утверждения. Рассмотрим случайную функцию, зависящую от двух переменных,

$$
\begin{aligned}
Z_{n}(t, s)= & \int_{0}^{t+} F_{n}(u-s) d L(u)=a \int_{0}^{t} F_{n}(u-s) d B(u) \\
& +b \int_{0}^{t} F_{n}(u-s) d u+\int_{0}^{t+} \int_{\mathbf{R}} x F_{n}(u-s) \tilde{N}(d u d x)
\end{aligned}
$$

$t, s \in \mathbf{T}$, где $B, \tilde{N}$ и $F_{n}$ определены в (4) и $(7)$ соответственно.

Приведем очевидные свойства данной функции.

Лемма 1. Функция $Z_{n}(t, s)$ обладает следующими свойствами:

1) при фиксированных $s \in \mathbf{T} u n \in \mathbf{N}$ прочесс $Z_{n}(t, s)$ является квадратично интегрируемьм $\mathscr{F}_{t}$-семимартингалом $[8$, гл. $2, \S 4]$;

2) $Z_{n}(t, s)=Z_{n}(s+1 / n, s)=L_{n}(s)$, если $t \geqslant s+1 / n$

3) $Z_{n}(t, s)=L(t)$ npu $t \leqslant s$.

Через $p$ обозначим целую часть дроби $1 /\left(n h_{n}\right)$, т.е. $p=\left[1 /\left(n h_{n}\right)\right]$, а

$$
[s]\left(h_{n}\right)=\tau_{t}+k h_{n}, \quad \text { если } \tau_{t}+k h_{n} \leqslant s<\tau_{t}+(k+1) h_{n} .
$$

Лемма 2. Пусть функиия $Z_{n}(t, s)$ определена формулой (11), последовательность случайных функиий $g_{n}(s, j)=g_{n}(s, j, \omega), s \in \mathbf{T}$, $j=0,1, \ldots, p+1$, такова, что $\sup _{s \in \mathbf{T}}\left|g_{n}(s, j)\right| \leqslant M$ для всех $n \in \mathbf{N}$ u $j=0,1, \ldots, p+1$ и почти всех $\omega \in \Omega$. Тогда справедливо следуюшее неравенство:

$$
\begin{aligned}
& \mathrm{E}\left(\sum_{j=0}^{p+1} g_{n}(s, j)\left(Z_{n}\left(s,[s]\left(h_{n}\right)-(j-1) h_{n}\right)-Z_{n}\left(s,[s]\left(h_{n}\right)-j h_{n}\right)\right)\right)^{2} \\
& \quad \leqslant \frac{C}{n}+C h_{n}+\frac{C}{n^{2} h_{n}} .
\end{aligned}
$$

Здесь и всюду в дальнейшем константы $C$ - абсолютные, т.е. не зависят от $s, t, n$ и случайной переменной $\omega \in \Omega$.

Д о к а з а т е л ь с т в о. Используя определение $Z_{n}(t, s)$ из $(11)$ и ее свойства из леммы 1 , получаем

$$
\begin{aligned}
& \mathbf{E}\left(\sum_{j=0}^{p+1} g_{n}(s, j)\left(Z_{n}\left(s,[s]\left(h_{n}\right)-(j-1) h_{n}\right)-Z_{n}\left(s,[s]\left(h_{n}\right)-j h_{n}\right)\right)\right)^{2} \\
& \quad \leqslant C(p+2) \sum_{j=0}^{p+1} \mathbf{E}\left(Z_{n}\left(s,[s]\left(h_{n}\right)-(j-1) h_{n}\right)-Z_{n}\left(s,[s]\left(h_{n}\right)-j h_{n}\right)\right)^{2}
\end{aligned}
$$




$$
\begin{aligned}
& \leqslant C(p+2) \sum_{j=0}^{p+1} \int_{0}^{s}\left(F_{n}\left(u-[s]\left(h_{n}\right)+(j-1) h_{n}\right)\right. \\
& \left.\quad-F_{n}\left(u-[s]\left(h_{n}\right)+j h_{n}\right)\right)^{2} d u \\
& \leqslant C(p+2) \int_{s-1 / n-2 h_{n}}^{s}\left(F_{n}\left(u-[s]\left(h_{n}\right)-h_{n}\right)\right. \\
& \left.\quad-F_{n}\left(u-[s]\left(h_{n}\right)+(p+1) h_{n}\right)\right) d u \\
& \leqslant C(p+2)\left(\frac{1}{n}+2 h_{n}\right) \leqslant C h_{n}+\frac{C}{n}+\frac{C}{n^{2} h_{n}},
\end{aligned}
$$

где промежуток интегрирования уменьшился из-за того, что из свойств функции $F_{n}$ вытекает, что

$$
F_{n}\left(u-[s]\left(h_{n}\right)-h_{n}\right)-F_{n}\left(u-[s]\left(h_{n}\right)+(p+1) h_{n}\right)=0 \quad \text { при } \quad u<s-\frac{1}{n}-2 h_{n} .
$$

Лемма 2 доказана.

Лемма 3. Если случайная функция $Z_{n}(t, s)$ определена в $(11)$, a $f \in \mathbf{C}_{B}^{2}(\mathbf{R}), m o$

$$
\begin{gathered}
\sup _{t \in \mathbf{T}} \mathbf{E}\left(\int_{\tau_{t}}^{t+1 / n} \int_{(s-1 / n) \vee \tau_{t}}^{s \wedge t} f_{n}\left(Z_{n}\left(s,[u]\left(h_{n}\right)\right)\right) \rho_{n}(s-u) d u d B(s)\right. \\
\left.-\int_{0}^{t} f(L(s)) d B(s)\right)^{2} \leqslant \frac{C}{n}+C h_{n} .
\end{gathered}
$$

Доказательство данной леммы проводится стандартными методами с использованием свойств интеграла Ито и вида функций $f_{n}, F_{n}$ и $Z_{n}$.

Введем следующее обозначение:

$$
\Theta_{n}\left(h_{n}\right)=\frac{1}{h_{n}} \int_{0}^{h_{n}} \int_{0}^{1 / n} F_{n}\left(u+\left\{\frac{s-u}{h_{n}}\right\} h_{n}\right) \rho_{n}(u) d u d s
$$

здесь и далее фигурные скобки обозначают дробную часть числа.

Очевидно, что $0 \leqslant \Theta_{n}\left(h_{n}\right) \leqslant 1$.

Лемма 4. Предположим, что $f \in \mathbf{C}_{B}^{1}(\mathbf{R})$, a $Z_{n} u F_{n}-u з$ (11) и (7) соответственно, тогда имеет место следуюшее неравенство:

$$
\begin{gathered}
\sup _{t \in \mathbf{T}} \mathbf{E}\left(\int_{\tau_{t}}^{t+1 / n} \int_{(s-1 / n) \vee \tau_{t}}^{s \wedge t} f_{n}\left(Z_{n}\left(s,[u]\left(h_{n}\right)\right)\right) F_{n}\left(s-[u]\left(h_{n}\right)\right) d F_{n}(s-u) d s\right. \\
\left.-\Theta_{n}\left(h_{n}\right) \int_{0}^{t} f(L(s)) d s\right)^{2} \leqslant \frac{C}{n}+C h_{n} .
\end{gathered}
$$

Лемма 5. Для любой Функции $f \in \mathbf{C}_{B}^{2}(\mathbf{R})$ справедливо неравенство

$$
\begin{gathered}
\sup _{t \in \mathbf{T}} \mathbf{E}\left(\int_{\tau_{t}}^{t+1 / n} \int_{\mathbf{R}} \int_{(s-1 / n) \vee \tau_{t}}^{s \wedge t} \quad x f_{n}\left(Z_{n}\left(s-,[u]\left(h_{n}\right)\right)+x F_{n}\left(s-[u]\left(h_{n}\right)\right)\right)\right. \\
\times \rho_{n}(s-u) d u \tilde{N}(d s d x)
\end{gathered}
$$




$$
\begin{array}{rl}
-\int_{0}^{t+} \int_{\mathbf{R}} \int_{0}^{1 / n} x & f\left(L(s-)+x F_{n}\left(u+\left\{\frac{s-u-t}{h_{n}}\right\} h_{n}\right)\right) \\
& \left.\times \rho_{n}(u) d u \tilde{N}(d s d x)\right)^{2} \leqslant \frac{C}{n}+C h_{n} .
\end{array}
$$

Доказательство лемм 4 и 5 проводится по той же схеме, что и в лемме 2. При этом используются неравенство Гёльдера, формула конечных приращений и свойства функций $f, Z_{n}, F_{n}$.

Введем функцию $G_{n}(u, s), u \in[0 ; 1], s \in \mathbf{T}$, следующим образом:

$$
G_{n}(u, s)=\left\{\begin{array}{c}
0, \quad u=0, \\
F_{n}\left((k-1) h_{n}+\left\{\frac{s}{h_{n}}\right\} h_{n}\right), \\
u \in\left(F_{n}\left(k h_{n}+\left\{\frac{s}{h_{n}}\right\} h_{n}\right) ;\right. \\
\left.F_{n}\left((k-1) h_{n}+\left\{\frac{s}{h_{n}}\right\} h_{n}\right)\right], \\
k=0,1, \ldots,\left[\frac{1}{n h_{n}}\right]+1 .
\end{array}\right.
$$

Напомним, что фигурные и квадратные скобки обозначают дробную и целую часть числа соответственно.

Для каждого $s \in \mathrm{T}$ функция $G_{n}(u, s)$ порождает вероятностную меру $\mu_{n}(d u, s)$ на борелевской $\sigma$-алгебре отрезка $[0 ; 1]$.

Учитывая (14) и определение (13) последовательности $\Theta_{n}\left(h_{n}\right)$, несложно получить, что

$$
\Theta_{n}\left(h_{n}\right)=\frac{1}{h_{n}} \int_{0}^{h_{n}} \int_{0}^{1} u \mu_{n}(d u, s) d s .
$$

Лемма 6. Последовательность мер $\mu_{n}(d u,-s), s \in \mathbf{T}$, порожденных функииями $G_{n}(u,-s), u \in[0 ; 1], s \in \mathbf{T}$, из (14), сходится слабо u в $\mathbf{L}^{1}(\mathbf{T})$ по переменной $s$ nрu $n \rightarrow \infty, h_{n} \rightarrow 0$ тогда $u$ только тогда, когда для каждого $\delta \in(0 ; 1)$ последовательность функиий $F_{n}\left(F_{n}^{-1}(u)-\delta h_{n}\right), u \in[0 ; 1]$, слабо сходится прu $n \rightarrow \infty, h_{n} \rightarrow 0 u$ предел не зависит от $\delta$.

Д о к а з а т е л с т в о. Покажем сначала, что если последовательность мер $\mu_{n}(d u,-s)$ сходится, то предельная мера не зависит от переменной $s$.

Итак, пусть последовательность мер $\mu_{n}(d u,-s)$ сходится в требуемом смысле к мере $\mu(d u,-s)$, тогда последовательность мер $\mu_{n}(d u,-s) d s$ на множестве $[0 ; 1] \times \mathbf{T}$ сходится слабо к мере $\mu(d u,-s) d s$. Таким образом, если $f(u, s) \in \mathbf{C}([0 ; 1] \times \mathbf{T})$, то при $n \rightarrow \infty, h_{n} \rightarrow 0$

$$
\int_{\mathbf{T}} \int_{0}^{1} f(u, s) \mu_{n}(d u,-s) d s \longrightarrow \int_{\mathbf{T}} \int_{0}^{1} f(u, s) \mu(d u,-s) d s .
$$


Представим левую часть (16) в виде суммы интегралов по отрезкам длины $h_{n}\left(\right.$ их будет $\left.m=\left[T / h_{n}\right]\right)$, а затем сделаем замену переменных и воспользуемся периодичностью по переменной $s$ меры $\mu_{n}(d u,-s)$ :

$$
\begin{aligned}
\int_{\mathbf{T}} \int_{0}^{1} f(u, s) \mu_{n}(d u,-s) d s= & \frac{1}{h_{n}} \int_{0}^{h_{n}} \int_{0}^{1} \sum_{k=0}^{m-1} f\left(u, s+k h_{n}\right) h_{n} \mu_{n}(d u,-s) d s \\
& +\int_{m h_{n}}^{T} \int_{0}^{1} f(u, s) \mu_{n}(d u,-s) d s .
\end{aligned}
$$

Очевидно, что последнее слагаемое стремится к нулю при $n \rightarrow \infty$, $h_{n} \rightarrow 0$. Кроме этого, по определению интеграла, $\sum_{k=0}^{m-1} f(u, s+$ $\left.k h_{n}\right) h_{n} \longrightarrow \int_{\mathbf{T}} f(u, s) d s$. Поэтому сходимость интеграла от функции $f(u, s)$ по мере $\mu_{n}(d u,-s) d s$ равносильна сходимости следующей последовательности и их пределы совпадают:

$$
\frac{1}{h_{n}} \int_{0}^{h_{n}} \int_{0}^{1} \int_{\mathbf{T}} f(u, t) d t \mu_{n}(d u,-s) d s .
$$

Воспользуемся определением меры $\mu_{n}$ и тем, что $\operatorname{supp} \rho_{n} \subseteq[0 ; 1 / n]$, тогда после очевидных преобразований имеем

$$
\begin{aligned}
& \int_{0}^{h_{n}} \int_{0}^{1} \int_{\mathbf{T}} \frac{f(u, t)}{h_{n}} d t \mu_{n}(d u,-s) d s \\
& \quad=\int_{\mathbf{T}} \int_{0}^{1 / n} \frac{\rho_{n}(u)}{h_{n}} \int_{u}^{u+h_{n}} f\left(F_{n}(s), t\right) d s d u d t=\int_{0}^{1} \int_{\mathbf{T}} f(u, t) d t \nu_{n}(d u),
\end{aligned}
$$

где $\nu_{n}(d u)=\left(F_{n}\left(F_{n}^{-1}(u)-h_{n}\right)-u\right) / \rho_{n}\left(F_{n}^{-1}(u)\right) d u$.

Пусть функция $f(u, s)$ имеет вид $f(u, s)=f_{1}(u) f_{2}(s)$, тогда

$$
\begin{aligned}
\int_{\mathbf{T}} \int_{0}^{1} f_{1}(u) f_{2}(s) \mu_{n}(d u,-s) d s & =\int_{\mathbf{T}} f_{2}(t) d t \int_{0}^{1} f_{1}(u) \nu_{n}(d u) \\
& \longrightarrow \int_{\mathbf{T}} f_{2}(t) d t \int_{0}^{1} f_{1}(u) \nu(d u) .
\end{aligned}
$$

Сравнив пределы в (16) и (18), имеем для $f(u, s)=f_{1}(u) f_{2}(s)$

$$
\int_{\mathbf{T}} \int_{0}^{1} f(u, s) \mu(d u,-s) d s=\int_{\mathbf{T}} \int_{0}^{1} f(u, s) \nu(d u) d s .
$$

Стандартными методами показывается, что $\mu(d u,-s) d s=\nu(d u) d s$, а значит, $\mu(d u,-s)=\nu(d u)$, т.е. предел последовательности мер $\mu_{n}(d u,-s)$ не зависит от переменной $s$.

Проведя аналогичные рассуждения, как и в случае, когда последовательность мер не зависит от параметра, можно доказать, что сходимость в требуемом смысле мер $\mu_{n}(d u,-s)$ равносильна слабой сходимости функций $G_{n}(u,-s)$, порождающих данные меры, по переменной $u \in[0 ; 1]$ и в $\mathbf{L}^{1}(\mathbf{T})$ по геременной $s$ при $n \rightarrow \infty, h_{n} \rightarrow 0$. 
Покажем, что слабая сходимость последовательности $F_{n}\left(F_{n}^{-1}(u)-\right.$ $\left.\delta h_{n}\right)$ по переменной $u \in[0 ; 1]$ для каждого $\delta \in(0 ; 1)$ при $n \rightarrow \infty, h_{n} \rightarrow 0$ и независимость предела от $\delta$ эквивалентны слабой сходимости функций $G_{n}(u,-s)$ по переменной $u \in[0 ; 1]$ и в $\mathbf{L}^{1}(\mathbf{T})$ по переменной $s$.

Несложными преобразованиями функции $G_{n}(u, s)$ из (14) получаем

$$
G_{n}(u,-s)=F_{n}\left(F_{n}^{-1}(u)-\left\{\frac{F_{n}^{-1}(u)+s}{h_{n}}\right\} h_{n}\right) .
$$

Как было доказано выше, предел последовательности $\mu_{n}(d u,-s)$ не зависит от переменной $s$, а значит, и предел $G_{n}(u,-s)$ не зависит от $s$. Рассмотрим возрастающую функцию $g(u), u \in[0 ; 1]$, и пусть $u-$ некоторая точка непрерывности этой функции. Тогда, если $\mathbf{T}=[0 ; T]$ и $m=\left[\left(T+F_{n}^{-1}(u)\right) / h_{n}\right]$, то

$$
\begin{aligned}
\int_{\mathbf{T}}\left|G_{n}(u,-s)-g(u)\right| d s=\int_{\mathbf{T}}\left|F_{n}\left(F_{n}^{-1}(u)-\left\{\frac{F_{n}^{-1}(u)+s}{h_{n}}\right\} h_{n}\right)-g(u)\right| d s \\
=h_{n} \int_{\left\{F_{n}^{-1}(u) / h_{n}\right\}}^{h_{n}}\left|F_{n}\left(F_{n}^{-1}(u)-s h_{n}\right)-g(u)\right| d s \\
\quad+h_{n} \int_{0}^{\left\{\left(T+F_{n}^{-1}(u)\right) / h_{n}\right\}}\left|F_{n}\left(F_{n}^{-1}(u)-s h_{n}\right)-g(u)\right| d s \\
\quad+\left(\left[\frac{T+F_{n}^{-1}(u)}{h_{n}}\right]-\left[\frac{F_{n}^{-1}(u)}{h_{n}}\right]-1\right) \\
\quad \times h_{n} \int_{0}^{1}\left|F_{n}\left(F_{n}^{-1}(u)-s h_{n}\right)-g(u)\right| d s=h_{n} J_{n}^{1}+h_{n} J_{n}^{2}+c_{n} h_{n} J_{n}^{3}, \quad(20)
\end{aligned}
$$

где $c_{n}=\left(\left[\left(T+F_{n}^{-1}(u)\right) / h_{n}\right]-\left[\left(F_{n}^{-1}(u)\right) / h_{n}\right]-1\right)$.

Очевидно, что $h_{n} J_{n}^{1} \rightarrow 0, h_{n} J_{n}^{2} \rightarrow 0$ и $c_{n} h_{n} \rightarrow T$ при $n \rightarrow \infty, h_{n} \rightarrow 0$. Значит, сходимость $G_{n}(u,-s)$ в требуемом смысле равносильна сходимости слагаемого $J_{n}^{3}$ из формулы $(20)$.

Заметим, что если $F_{n}\left(F_{n}^{-1}(u)-s h_{n}\right) \rightarrow g(u)$ для всех $s \in(0 ; 1)$, то по теореме Лебега о мажорируемой сходимости $J_{n}^{3} \rightarrow 0$ при $n \rightarrow \infty$, $h_{n} \rightarrow 0$, а значит, и $G_{n}(u,-s)$ сходится в требуемом смысле. Таким образом, для завершения доказательства леммы осталось показать, что сходимость $G_{n}(u,-s)$ влечет сходимость $F_{n}\left(F_{n}^{-1}(u)-s h_{n}\right)$.

Пусть $G_{n}(u,-s) \rightarrow g(u)$ в требуемом смысле. Заметим, что из определения функции $G_{n}(u,-s)$ вытекает неравенство $G_{n}(u,-s) \geqslant u$ для всех $s \in \mathbf{T}$, тогда и $g(u) \geqslant u, u \in[0 ; 1]$, а значит, и $F_{n}^{-1}(g(u)) \leqslant F_{n}^{-1}(u)$. Из $(20)$ вытекает, что $J_{n}^{3} \rightarrow 0$ при $n \rightarrow \infty, h_{n} \rightarrow 0$. Рассмотрим отдельно два случая:

$$
F_{n}^{-1}(g(u)) \leqslant F_{n}^{-1}(u)-h_{n} \quad \text { и } \quad F_{n}^{-1}(g(u))>F_{n}^{-1}(u)-h_{n} .
$$

Итак, пусть $F_{n}^{-1}(g(u)) \leqslant F_{n}^{-1}(u)-h_{n}$, тогда из определения (7) функ- 
ции $F_{n}$ получаем

$$
\begin{aligned}
J_{n}^{3} & =\int_{F_{n}^{-1}(g(u))}^{F_{n}^{-1}(u)-h_{n}} \rho_{n}(v) d v+\int_{0}^{1} \int_{F_{n}^{-1}(u)-h_{n}}^{F_{n}^{-1}(u)-s h_{n}} \rho_{n}(v) d v d s \\
& =g(u)-F_{n}\left(F_{n}^{-1}(u)-h_{n}\right)+\int_{F_{n}^{-1}(u)-h_{n}}^{F_{n}^{-1}(u)} \frac{F_{n}^{-1}(u)-v}{h_{n}} \rho_{n}(v) d v .
\end{aligned}
$$

Зафиксируем произвольное $\delta \in(0 ; 1)$ и оценим $J_{n}^{3}$ снизу:

$$
J_{n}^{3}=\left(g(u)-F_{n}\left(F_{n}^{-1}(u)-h_{n}\right)\right)+\delta\left(F_{n}\left(F_{n}^{-1}(u)-h_{n}\right)-F_{n}\left(F_{n}^{-1}(u)-\delta h_{n}\right)\right) \geqslant 0 .
$$

Так как $J_{n}^{3} \rightarrow 0$, то и левая часть последнего неравенства стремится к нулю, а значит, в силу неотрицательности, и каждое слагаемое стремится к нулю. А это означает, что $F_{n}\left(F_{n}^{-1}(u)-h_{n}\right) \rightarrow$ $g(u)$ и $F_{n}\left(F_{n}^{-1}(u)-h_{n}\right)-F_{n}\left(F_{n}^{-1}(u)-\delta h_{n}\right) \rightarrow 0$. Отсюда вытекает $F_{n}\left(F_{n}^{-1}(u)-\delta h_{n}\right) \rightarrow g(u)$ при $n \rightarrow \infty, h_{n} \rightarrow 0$ для всех $\delta \in(0 ; 1)$ и любой точки непрерывности $u$ функции $g(u)$.

Рассмотрим теперь второй случай, т.е.

$$
F_{n}^{-1}(g(u))>F_{n}^{-1}(u)-h_{n}
$$

тогда

$$
\begin{aligned}
J_{n}^{3}= & \int_{0}^{1}\left|\int_{F_{n}^{-1}(g(u))}^{F_{n}^{-1}(u)-s h_{n}} \rho_{n}(v) d v\right| d s=\int_{F_{n}^{-1}(g(u))}^{F_{n}^{-1}(u)} \frac{F_{n}^{-1}(u)-v}{h_{n}} \rho_{n}(v) d v \\
& +\int_{F_{n}^{-1}(u)-h_{n}}^{F_{n}^{-1}(g(u))}\left(1-\frac{F_{n}^{-1}(u)-v}{h_{n}}\right) \rho_{n}(v) d v .
\end{aligned}
$$

Зафиксируем произвольное $\delta \in(0 ; 1)$. В силу положительности интегралов в (21) получаем, что если $F_{n}^{-1}(u)-\delta h_{n}<F_{n}^{-1}(g(u))$, то

$$
\begin{aligned}
J_{n}^{3} & \geqslant \int_{F_{n}^{-1}(u)-h_{n}}^{F_{n}^{-1}(g(u))}\left(1-\frac{F_{n}^{-1}(u)-v}{h_{n}}\right) \rho_{n}(v) d v \\
& \geqslant(1-\delta)\left(F_{n}\left(F_{n}^{-1}(u)-\delta h_{n}\right)-g(u)\right) \geqslant 0 .
\end{aligned}
$$

Аналогично, для $F_{n}^{-1}(u)-\delta h_{n} \geqslant F_{n}^{-1}(g(u))$ имеем

$$
J_{n}^{3} \geqslant \int_{F_{n}^{-1}(g(u))}^{F_{n}^{-1}(u)} \frac{F_{n}^{-1}(u)-v}{h_{n}} \rho_{n}(v) d v \geqslant \delta\left(g(u)-F_{n}\left(F_{n}^{-1}(u)-\delta h_{n}\right)\right) \geqslant 0 .
$$

Объединяя оценки (21)-(23) и учитывая то, что $J_{n}^{3} \rightarrow 0$, получаем требуемое. Лемма 6 доказана.

3. Доказательство основных результатов. Доказательство предложения 1 получается после применения формулы Ито к интегральным суммам из определения 1 : 
Докажем теорему 1 . Для этого преобразуем сумму $S_{n} f(t)$, используя свойства функции $Z_{n}$, приведенные в лемме 1 :

$$
\begin{aligned}
& S_{n} f(t)=\sum_{k=0}^{m_{t}-1} f_{n}\left(Z_{n}\left(\tau_{t}+(k+p+2) h_{n}-, \tau_{t}+k h_{n}\right)\right) \\
& \times\left(Z_{n}\left(\tau_{t}+(k+p+2) h_{n}-, \tau_{t}+(k+1) h_{n}\right)\right. \\
& \left.-Z_{n}\left(\tau_{t}+(k+p+2) h_{n}-, \tau_{t}+k h_{n}\right)\right) \\
& =\sum_{j=0}^{p+1} \sum_{k=j}^{m_{t}+j-1}\left(f_{n}\left(Z_{n}\left(\tau_{t}+(k+1) h_{n}-, \tau_{t}+(k-j) h_{n}\right)\right)\right. \\
& \times\left(Z_{n}\left(\tau_{t}+(k+1) h_{n}-, \tau_{t}+(k-j+1) h_{n}\right)\right. \\
& \left.-Z_{n}\left(\tau_{t}+(k+1) h_{n}-, \tau_{t}+(k-j) h_{n}\right)\right) \\
& -f_{n}\left(Z_{n}\left(\tau_{t}+k h_{n}-, \tau_{t}+(k-j) h_{n}\right)\right) \\
& \times\left(Z_{n}\left(\tau_{t}+k h_{n}-, \tau_{t}+(k-j+1) h_{n}\right)\right. \\
& \left.\left.-Z_{n}\left(\tau_{t}+k h_{n}-, \tau_{t}+(k-j) h_{n}\right)\right)\right) \text {. }
\end{aligned}
$$

Так как из леммы 1 вытекает, что $Z_{n}(t, s)$ - семимартингал по первой переменной, то мы можем применить формулу Ито (см., например, $[8$, гл. $2, \S 5])$ к последнему выражению. Тогда

$$
\begin{aligned}
S_{n} f(t)= & a \sum_{j=0}^{p+1} \sum_{k=j}^{m_{t}+j-1} \int_{\tau_{t}+k h_{n}}^{\tau_{t}+(k+1) h_{n}} f_{n}^{\prime}\left(Z_{n}\left(s,[s]\left(h_{n}\right)-j h_{n}\right)\right) \\
& \times F_{n}\left(s-[s]\left(h_{n}\right)+j h_{n}\right) \\
& \times\left(Z_{n}\left(s,[s]\left(h_{n}\right)-(j-1) h_{n}\right)-Z_{n}\left(s,[s]\left(h_{n}\right)-j h_{n}\right)\right) d B(s) \\
+ & b \sum_{j=0}^{p+1} \sum_{k=j}^{m_{t}+j-1} \int_{\tau_{t}+k h_{n}}^{\tau_{t}+(k+1) h_{n}} f_{n}^{\prime}\left(Z_{n}\left(s,[s]\left(h_{n}\right)-j h_{n}\right)\right) \\
& \times\left(Z_{n}\left(s,[s]\left(h_{n}\right)-(j-1) h_{n}\right)-Z_{n}\left(s,[s]\left(h_{n}\right)-j h_{n}\right)\right) \\
+ & \quad a \sum_{j=0}^{p+1} \sum_{k=j}^{m_{t}+j-1}\left(s-[s]\left(h_{n}\right)+j h_{n}\right) d s \\
& \times\left(F_{n}\left(s-[s]\left(h_{n}\right)+(j-1) h_{n}\right)-F_{n}\left(s-[s]\left(h_{n}\right)+j h_{n}\right)\right) d B(s) \\
+ & b \sum_{j=0}^{p+1} \sum_{k=j}^{\tau_{t}+j-1} \int_{\tau_{t}+k h_{n}}^{\tau_{t}+(k+1) h_{n}} f_{n}\left(Z_{n}\left(s,[s]\left(h_{n}\right)-j h_{n}\right)\right) \\
& \times\left(F_{n}\left(s-[s]\left(h_{n}\right)+(j-1) h_{n}\right)-F_{n}\left(s-[s]\left(h_{n}\right)+j h_{n}\right)\right) d s
\end{aligned}
$$




$$
\begin{aligned}
& +\frac{a^{2}}{2} \sum_{j=0}^{p+1} \sum_{k=j}^{m_{t}+j-1} \int_{\tau_{t}+k h_{n}}^{\tau_{t}+(k+1) h_{n}} f_{n}^{\prime \prime}\left(Z_{n}\left(s,[s]\left(h_{n}\right)-j h_{n}\right)\right) \\
& \times F_{n}^{2}\left(s-[s]\left(h_{n}\right)+j h_{n}\right) \\
& \times\left(Z_{n}\left(s,[s]\left(h_{n}\right)-(j-1) h_{n}\right)-Z_{n}\left(s,[s]\left(h_{n}\right)-j h_{n}\right)\right) d s \\
& +a^{2} \sum_{j=0}^{p+1} \sum_{k=j}^{m_{t}+j-1} \int_{\tau_{t}+k h_{n}}^{\tau_{t}+(k+1) h_{n}} f_{n}^{\prime}\left(Z_{n}\left(s,[s]\left(h_{n}\right)-j h_{n}\right)\right) \\
& \times\left(F_{n}\left(s-[s]\left(h_{n}\right)+(j-1) h_{n}\right)-F_{n}\left(s-[s]\left(h_{n}\right)+j h_{n}\right)\right) \\
& \times F_{n}\left(s-[s]\left(h_{n}\right)+j h_{n}\right) d s \\
& +\sum_{j=0}^{p+1} \sum_{k=j}^{m_{t}+j-1} \int_{\tau_{t}+k h_{n}}^{\tau_{t}+(k+1) h_{n}} \int_{\mathbf{R}}\left(f _ { n } \left(Z_{n}\left(s-,[s]\left(h_{n}\right)-j h_{n}\right)\right.\right. \\
& \left.+x F_{n}\left(s-[s]\left(h_{n}\right)+j h_{n}\right)\right) \\
& \times\left(Z_{n}\left(s-,[s]\left(h_{n}\right)-(j-1) h_{n}\right)-Z_{n}\left(s-,[s]\left(h_{n}\right)-j h_{n}\right)\right. \\
& \left.+x\left(F_{n}\left(s-[s]\left(h_{\text {in }}\right)+(j-1) h_{n}\right)-F_{n}\left(s-[s]\left(h_{n}\right)+j h_{n}\right)\right)\right) \\
& -f_{n}\left(Z_{n}\left(s-,[s]\left(h_{n}\right)-j h_{n}\right)\right) \\
& \times\left(Z_{n}\left(s-,[s]\left(h_{n}\right)-(j-1) h_{n}\right)\right. \\
& \left.\left.-Z_{n}\left(s-,[s]\left(h_{n}\right)-j h_{n}\right)\right)\right) \tilde{N}(d s d x) \\
& +\sum_{j=0}^{p+1} \sum_{k=j}^{m_{t}+j-1} \int_{\tau_{t}+k h_{n}}^{\tau_{t}+(k+1) h_{n}} \int_{\mathbf{R}}\left(f _ { n } \left(Z_{n}\left(s,[s]\left(h_{n}\right)-j h_{n}\right)\right.\right. \\
& \left.+x F_{n}\left(s-[s]\left(h_{n}\right)+j h_{n}\right)\right) \\
& \times\left(Z_{n}\left(s,[s]\left(h_{n}\right)-(j-1) h_{n}\right)-Z_{n}\left(s,[s]\left(h_{n}\right)-j h_{n}\right)\right. \\
& \left.+x\left(F_{n}\left(s-[s]\left(h_{n}\right)+(j-1) h_{n}\right)-F_{n}\left(s-[s]\left(h_{n}\right)+j h_{n}\right)\right)\right) \\
& -f_{n}\left(Z_{n}\left(s,[s]\left(h_{n}\right)-j h_{n}\right)\right) \\
& \times\left(Z_{n}\left(s,[s]\left(h_{n}\right)-(j-1) h_{n}\right)-Z_{n}\left(s,[s]\left(h_{n}\right)-j h_{n}\right)\right) \\
& -x f_{n}^{\prime}\left(Z_{n}\left(s,[s]\left(h_{n}\right)-j h_{n}\right)\right) \\
& \times\left(Z_{n}\left(s,[s]\left(h_{n}\right)-(j-1) h_{n}\right)-Z_{n}\left(s,[s]\left(h_{n}\right)-j h_{n}\right)\right) \\
& \times F_{n}\left(s-[s]\left(h_{n}\right)+j h_{n}\right)-x f_{n}\left(Z_{n}\left(s,[s]\left(h_{n}\right)-j h_{n}\right)\right) \\
& \times\left(F_{n}\left(s-[s]\left(h_{n}\right)+(j-1) h_{n}\right)\right. \\
& \left.\left.-F_{n}\left(s-[s]\left(h_{n}\right)+j h_{n}\right)\right)\right) \widehat{N}(d s d x) \\
& =a I_{1}(t)+b I_{2}(t)+a I_{3}(t)+b I_{4}(t)+\frac{a^{2}}{2} I_{5}(t) \\
& +a^{2} I_{6}(t)+I_{7}(t)+I_{8}(t) \text {. }
\end{aligned}
$$


Рассмотрим $I_{1}(t)$. Воспользуемся аддитивностью интеграла, а затем внесем знак суммы под интеграл:

$$
\begin{aligned}
I_{1}(t)=\int_{\tau_{t}}^{t+(p+1)} & \sum_{j=0}^{h_{n}} I_{\left[\tau_{t}+j h_{n} ; t+j h_{n}\right]}^{p+1}(s) f_{n}^{\prime}\left(Z_{n}\left(s,[s]\left(h_{n}\right)-j h_{n}\right)\right) \\
& \times F_{n}\left(s-[s]\left(h_{n}\right)+j h_{n}\right) \\
& \times\left(Z_{n}\left(s,[s]\left(h_{n}\right)-(j-1) h_{n}\right)-Z_{n}\left(s,[s]\left(h_{n}\right)-j h_{n}\right)\right) d B(s),
\end{aligned}
$$

здесь $I_{\left[\tau_{t}+j h_{n} ; t+j h_{n}\right]}(s)$ - индикаторная функция отрезка $\left[\tau_{t}+j h_{n} ; t+j h_{n}\right]$.

Оценим математическое ожидание квадрата последнего выражения. Из свойств интеграла Ито имеем

$$
\begin{array}{rl}
\sup _{t \in \mathbf{T}} & \mathbf{E}\left(I_{1}(t)\right)^{2} \\
=\sup _{t \in \mathbf{T}} \int_{\tau_{t}}^{t+(p+1) h_{n}} \mathbf{E}\left(\sum_{j=0}^{p+1} I_{\left[\tau_{t}+j h_{n} ; t+j h_{n}\right]}(s) F_{n}\left(s-[s]\left(h_{n}\right)+j h_{n}\right)\right. \\
\quad \times f_{n}^{\prime}\left(Z_{n}\left(s,[s]\left(h_{n}\right)-j h_{n}\right)\right)
\end{array}
$$

где последнее неравенство вытекает из леммы 2 , если положить

$$
g_{n}(s, j)=I_{\left[\tau_{t}+j h_{n} ; t+j h_{n}\right]}(s) F_{n}\left(s-[s]\left(h_{n}\right)+j h_{n}\right) f_{n}^{\prime}\left(Z_{n}\left(s,[s]\left(h_{n}\right)-j h_{n}\right)\right) .
$$

Аналогичным образом для $I_{2}(t)$ с помощью неравенства Гёльдера и леммы 2 можно получить

$$
\sup _{t \in \mathbf{T}} \mathbf{E}\left(I_{2}(t)\right)^{2} \leqslant \frac{C}{n}+C h_{n}+\frac{C}{n^{2} h_{n}} .
$$

Применив такую же технику, как и выше, а также лемму 2 при

$$
g_{n}(s, j)=I_{\left[\tau_{t}+j h_{n} ; t+j h_{n}\right]}(s) F_{n}^{2}\left(s-[s]\left(h_{n}\right)+j h_{n}\right) f_{n}^{\prime \prime}\left(Z_{n}\left(s,[s]\left(h_{n}\right)-j h_{n}\right)\right)
$$

к слагаемому $I_{5}(t)$, имеем

$$
\sup _{t \in \mathbf{T}} \mathbf{E}\left(I_{5}(t)\right)^{2} \leqslant \frac{C}{n}+C h_{n}+\frac{C}{n^{2} h_{n}} .
$$

Преобразуем $I_{3}(t)$. Для этого воспользуемся определением $F_{n}$ из $(7)$ затем внесем сумму под интеграл и по определению $[s]\left(h_{n}\right)$ получим $I_{3}(t)=\int_{\tau_{t}}^{t+(p+1) h_{n}} \int_{\left(\left([s]\left(h_{n}\right)-(p+1) h_{n}\right) \vee \tau_{t}\right) \wedge t}^{\left([s]\left(h_{n}\right)+h_{n}\right) \wedge t} f_{n}\left(Z_{n}\left(s,[u]\left(h_{n}\right)\right)\right) \rho_{n}(s-u) d u d B(s)$. 
Заметим, что из того, что $\operatorname{supp} \rho_{n} \subseteq[0 ; 1 / n]$, вытекает, что $\rho_{n}(s-$ $u)=0$, если $s \leqslant u$ или $u \leqslant s-1 / n$. Поэтому, так как $[s]\left(h_{n}\right)+h_{n} \geqslant s$ и $[s]\left(h_{n}\right)-(p+1) h_{n} \leqslant s-1 / n$, то во внутреннем интеграле пределы интегрирования можно заменить на $s \wedge t$ и $(s-1 / n) \vee \tau_{t}$ соответственно. Кроме этого, во внешнем интеграле верхний предел можно положить равным $t+1 / n<t+(p+1) h_{n}$, так как при $t+(p+1) h_{n}>s>t+$ $1 / n$ пределы внутреннего интеграла будут равны $t$. Учитывая все это, получаем

$$
I_{3}(t)=\int_{\tau_{t}}^{t+1 / n} \int_{(s-1 / n) \vee \tau_{t}}^{s \wedge t} f_{n}\left(Z_{n}\left(s,[u]\left(h_{n}\right)\right)\right) \rho_{n}(s-u) d u d B(s) .
$$

Для $I_{4}(t)$, поступая подобным образом, имеем

$$
I_{4}(t)=\int_{\tau_{t}}^{t+1 / n} \int_{(s-1 / n) \vee \tau_{t}}^{s \wedge t} f_{n}\left(Z_{n}\left(s,[u]\left(h_{n}\right)\right)\right) \rho_{n}(s-u) d u d s .
$$

Поэтому из леммы 3 следует

$$
\begin{gathered}
\sup _{t \in \mathbf{T}} \mathbf{E}\left(I_{3}(t)-\int_{0}^{t} f(L(s)) d B(s)\right)^{2} \leqslant \frac{C}{n}+C h_{n}, \\
\sup _{t \in \mathbf{T}} \mathbf{E}\left(I_{4}(t)-\int_{0}^{t} f(L(s)) d s\right)^{2} \leqslant \frac{C}{n}+C h_{n} .
\end{gathered}
$$

Применяя вышеуказанную технику к $I_{6}(t)$, несложно получить $I_{6}(t)=\int_{\tau_{t}}^{t+1 / n} \int_{(s-1 / n) \vee \tau_{t}}^{s \wedge t} f_{n}^{\prime}\left(Z_{n}\left(s,[u]\left(h_{n}\right)\right)\right) F_{n}\left(s-[u]\left(h_{n}\right)\right) \rho_{n}(s-u) d u d s$.

Непосредственно из леммы 4 видно, что

$$
\sup _{t \in \mathbf{T}} \mathbf{E}\left(I_{6}(t)-\Theta_{n}\left(h_{n}\right) \int_{0}^{t} f^{\prime}(L(s)) d s\right)^{2} \leqslant \frac{C}{n}+C h_{n},
$$

где $\Theta_{n}\left(h_{n}\right)$ определено в (13).

Выражение $I_{7}(t)$ представим в виде двух слагаемых:

$$
\begin{aligned}
I_{7}(t)= & \sum_{j=0}^{p+1} \int_{\tau_{t}+j h_{n}}^{t+j h_{n}} \int_{\mathbf{R}}\left(f_{n}\left(Z_{n}\left(s-,[s]\left(h_{n}\right)-j h_{n}\right)+x F_{n}\left(s-[s]\left(h_{n}\right)+j h_{n}\right)\right)\right. \\
& \left.-f_{n}\left(Z_{n}\left(s-,[s]\left(h_{n}\right)-j h_{n}\right)\right)\right) \\
& \times\left(Z_{n}\left(s-,[s]\left(h_{n}\right)-(j-1) h_{n}\right)-Z_{n}\left(s-,[s]\left(h_{n}\right)-j h_{n}\right)\right) \tilde{N}(d s d x) \\
& +\sum_{j=0}^{p+1} \int_{\tau_{t}+j h_{n}}^{t+j h_{n}} \int_{\mathbf{R}} x f_{n}\left(Z_{n}\left(s-,[s]\left(h_{n}\right)-j h_{n}\right)\right. \\
& \left.+x F_{n}\left(s-[s]\left(h_{n}\right)+j h_{n}\right)\right)
\end{aligned}
$$




$$
\begin{aligned}
& \times\left(F_{n}\left(s-[s]\left(h_{n}\right)+(j-1) h_{n}\right)-F_{n}\left(s-[s]\left(h_{n}\right)+j h_{n}\right)\right) \tilde{N}(d s d x) \\
= & I_{71}(t)+I_{72}(t) .
\end{aligned}
$$

Как и для $I_{1}(t)$, несложно получить

$$
\begin{aligned}
I_{71}(t)= & \int_{\tau_{t}}^{t+(p+2) h_{n}} \int_{\mathbf{R}} \sum_{j=0}^{p+1} I_{\left[\tau_{t}+j h_{n} ; t+j h_{n}\right]}(s)\left(f _ { n } \left(Z_{n}\left(s-,[s]\left(h_{n}\right)-j h_{n}\right)\right.\right. \\
& \left.\left.+x F_{n}\left(s-[s]\left(h_{n}\right)+j h_{n}\right)\right)-f_{n}\left(Z_{n}\left(s-,[s]\left(h_{n}\right)-j h_{n}\right)\right)\right) \\
& \times\left(Z_{n}\left(s-,[s]\left(h_{n}\right)-(j-1) h_{n}\right)-Z_{n}\left(s-,[s]\left(h_{n}\right)-j h_{n}\right)\right) \tilde{N}(d s d x) .
\end{aligned}
$$

Из этого представления в силу свойств стохастического интеграла Ито и леммы 2 легко вытекает оценка для математического ожидания квадрата $I_{71}(t)$ :

$$
\sup _{t \in \mathbf{T}} \mathbf{E}\left(I_{71}(t)\right)^{2} \leqslant \frac{C}{n}+C h_{n}+\frac{C}{n^{2} h_{n}} .
$$

Рассмотрим $I_{72}(t)$. Применяя такие же преобразования, как и для $I_{3}(t)$, имеем

$$
\begin{array}{rl}
I_{72}(t)=\int_{\tau_{t}}^{t+1 / n} \int_{\mathbf{R}} \int_{(s-1 / n) \vee \tau_{t}}^{s \wedge t} & x f_{n}\left(Z_{n}\left(s-,[u]\left(h_{n}\right)\right)+x F_{n}\left(s-[u]\left(h_{n}\right)\right)\right) \\
& \times \rho_{n}(s-u) d u \tilde{N}(d s d x) .
\end{array}
$$

Для последнего выражения из леммы 5 вытекает оценка

$$
\begin{aligned}
\sup _{t \in \mathbf{T}} \mathrm{E}\left(I_{72}(t)-\int_{0}^{t+} \int_{\mathbf{R}} \int_{0}^{1 / n} x f\right. & \left(L(s-)+x F_{n}\left(u+\left\{\frac{s-u-t}{h_{n}}\right\} h_{n}\right)\right) \\
& \left.\times \rho_{n}(u) d u \tilde{N}(d s d x)\right)^{2} \leqslant \frac{C}{n}+C h_{n} .
\end{aligned}
$$

Объединяя оценки (30) и (31), получаем

$$
\begin{aligned}
\sup _{t \in \mathbf{T}} \mathbf{E}\left(I_{7}(t)-\int_{0}^{t+}\right. & \int_{\mathbf{R}} \int_{0}^{1 / n} x f\left(L(s-)+x F_{n}\left(u+\left\{\frac{s-u-t}{h_{n}}\right\} h_{n}\right)\right) \\
& \left.\times \rho_{n}(u) d u \tilde{N}(d s d x)\right)^{2} \leqslant \frac{C}{n}+C h_{n}+\frac{C}{n^{2} h_{n}} .
\end{aligned}
$$

Для исследования $I_{8}(t)$ применим технику, аналогичную использованной при оценивании $I_{7}(t)$. Тогда получим следующее неравенство:

$$
\begin{gathered}
\sup _{t \in \mathbf{T}} \mathbf{E}\left(I_{8}(t)-\int_{0}^{t+} \int_{\mathbf{R}} x\left(\int_{0}^{1 / n} f\left(L(s)+x F_{n}\left(u+\left\{\frac{s-u-t}{h_{n}}\right\} h_{n}\right)\right)\right.\right. \\
\left.\left.\times \rho_{n}(u) d u-f(L(s))\right) \widehat{N}(d s d x)\right)^{2} \\
\leqslant \frac{C}{n}+C h_{n}+\frac{C}{n^{2} h_{n}} .
\end{gathered}
$$


В итоге неравенства (24)-(29), (32) и (33) влекут

$$
\begin{aligned}
\sup _{t \in \mathbf{T}} \mathbf{E} & \left(S_{n} f(t)-a \int_{0}^{t} f(L(s)) d B(s)-b \int_{0}^{t} f(L(s)) d s\right. \\
& -a^{2} \Theta_{n}\left(h_{n}\right) \int_{0}^{t} f^{\prime}(L(s)) d s \\
- & \int_{0}^{t+} \int_{\mathbf{R}} \int_{0}^{1 / n} x f\left(L(s-)+x F_{n}\left(u+\left\{\frac{s-u-t}{h_{n}}\right\} h_{n}\right)\right) \\
- & \int_{0}^{t+} \int_{\mathbf{R}} x\left(\int_{0}^{1 / n} f\left(L(s)+x F_{n}\left(u+\left\{\frac{s-u-t}{h_{n}}\right\} h_{n}\right)\right) \rho_{n}(u) d u\right. \\
& \quad-f(L(s))) \widehat{N}(d s d x))^{2} \leqslant \frac{C}{n}+C h_{n}+\frac{C}{n^{2} h_{n}} .
\end{aligned}
$$

С помощью мер $\mu_{n}(d u, s)$, порожденных функциями $G_{n}(u, s)$ из $(14)$, формула (34) запишется таким образом:

$$
\begin{aligned}
\sup _{t \in \mathbf{T}} \mathbf{E} & \left(S_{n} f(t)-a \int_{0}^{t} f(L(s)) d B(s)-b \int_{0}^{t} f(L(s)) d s\right. \\
& -a^{2} \Theta_{n}\left(h_{n}\right) \int_{0}^{t} f^{\prime}(L(s)) d s \\
& -\int_{0}^{t+} \int_{\mathbf{R}} \int_{0}^{1 / n} x f(L(s-)+x u) \mu_{n}(d u, s-t) \tilde{N}(d s d x) \\
& \left.-\int_{0}^{t+} \int_{\mathbf{R}} x\left(\int_{0}^{1 / n} f(L(s)+x u) \mu_{n}(d u, s-t)-f(L(s))\right) \widehat{N}(d s d x)\right)^{2} \\
\leqslant & \frac{C}{n}+C h_{n}+\frac{C}{n^{2} h_{n}} .
\end{aligned}
$$

Из последнего неравенства видно, что сходимость $S_{n} f(t)$ равносильна сходимости суммы последних трех слагаемых под знаком математического ожидания в (35). А сходимость этой суммы, как нетрудно установить, эквивалентна слабой сходимости последовательности мер $\mu_{n}(d u,-s)$ в $\mathbf{L}^{1}(\mathbf{T})$ по переменной $s$. Но, как было доказано в лемме 6 , слабая сходимость данной последовательности мер в этом смысле равносильна слабой сходимости (т.е. в каждой точке непрерывности предельной функции) для каждого $\delta \in(0 ; 1)$ последовательности функций $F_{n}\left(F_{n}^{-1}(u)-\delta h_{n}\right), u \in[0 ; 1]$, при $n \rightarrow \infty, h_{n} \rightarrow 0$ и независимости предела от $\delta$. Теорема 1 доказана.

Доказательство теоремы 2. Пусть для каждого $\delta \in(0 ; 1)$ последовательность функций $F_{n}\left(F_{n}^{-1}(u)-\delta h_{n}\right)$ слабо сходится к $g(u), u \in[0 ; 1]$, при $n \rightarrow \infty, h_{n} \rightarrow 0$. Так как $F_{n}\left(F_{n}^{-1}(u)-\delta h_{n}\right) \geqslant u$, то и $g(u) \geqslant u$. Покажем, что если $v-$ точка непрерывности и $g(v)=d>v$, то $g(u)=d$ для всех $u \in[v ; d]$. Откуда и будет следовать, что $g(u)$ из класса $\bar{G}$. 
Возьмем $1>\varepsilon>\delta>0$, тогда из условия теоремы 2 вытекает, что $F_{n}\left(F_{n}^{-1}(v)-(\varepsilon-\delta) h_{n}\right) \rightarrow g(v)$. Поэтому для произвольного фиксированного $u \in(v ; d)$ и достаточно больших $n$

$$
v<u<F_{n}\left(F_{n}^{-1}(v)-(\varepsilon-\delta) h_{n}\right) .
$$

Откуда

$$
\begin{aligned}
F_{n}^{-1}(v) & \geqslant F_{n}^{-1}(u) \geqslant F_{n}^{-1}(v)-(\varepsilon-\delta) h_{n}, \\
F_{n}\left(F_{n}^{-1}(v)-\delta h_{n}\right) & \leqslant F_{n}\left(F_{n}^{-1}(u)-\delta h_{n}\right) \leqslant F_{n}\left(F_{n}^{-1}(v)-\varepsilon h_{n}\right) .
\end{aligned}
$$

Переходя в последнем неравенстве к пределу при $n \rightarrow \infty, h_{n} \rightarrow 0$ и учитывая, что $F_{n}\left(F_{n}^{-1}(v)-\delta h_{n}\right) \rightarrow g(v)$ и $F_{n}\left(F_{n}^{-1}(v)-\varepsilon h_{n}\right) \rightarrow g(v)$, получаем $F_{n}\left(F_{n}^{-1}(u)-\delta h_{n}\right) \rightarrow g(v)=d$, а значит, $g(u)=g(v)=d$.

Для доказательства того, что $S_{n} f(t) \rightarrow(\mu) \int_{0}^{t+} f(L(s-)) d L(s)$, перейдем к пределу при $n \rightarrow \infty, h_{n} \rightarrow 0$ в неравенстве (35) и учтем, что предельная мера $\mu(d u)$ порождена функцией $g(u)$. Теорема 2 доказана.

Д ок а з а т е л ь с т в о с л е д с т в и я 1. Пусть последовательность «шапочек» $\rho_{n}$ имеет вид $\rho_{n}(t)=n \rho(n t)$. Тогда $F_{n}\left(F_{n}^{-1}(u)-\delta h_{n}\right)=$ $F\left(F^{-1}(u)-\delta n h_{n}\right)$, где $F(t)=\int_{t}^{1} \rho(s) d s$ и $F^{-1}(s)=\sup \{u: F(u)=s\}$. Поэтому сходимость и независимость предела от $\delta$ имеет место только в случаях, когда $h_{n}=o(1 / n)$ либо $1 / n=o\left(h_{n}\right)$. Применяя теоремы 1 и 2 , получаем следствие 1 , причем в данном случае оценки теоремы 1 могут быть улучшены, что позволяет отказаться от условия $n^{2} h_{n} \rightarrow \infty$.

Для доказательства следствия 2 заметим, что при непрерывном процессе $L(t)$ в неравенстве (35) будут отсутствовать последние два слагаемых, поэтому сходимость сумм $S_{n} f(t)$ эквивалентна сходимости последовательности $\Theta_{n}\left(h_{n}\right)$ из (13). Найдем связь между $K\left(n ; h_{n}\right)$ и $\Theta_{n}\left(h_{n}\right)$, используя те же преобразования, что и для вывода формул (15) и (17):

$$
\begin{aligned}
K\left(n ; h_{n}\right) & =-2 \int_{0}^{1 / n} \int_{s-h_{n}}^{s}\left(1-\frac{s-\tau}{h_{n}}\right) \rho_{n}(\tau) d \tau d F_{n}(s) \\
& =2 \int_{0}^{1 / n} F_{n}(s) \rho_{n}(s) d s-\frac{2}{h_{n}} \int_{0}^{1 / n} F_{n}(s) \int_{s-h_{n}}^{s} \rho_{n}(\tau) d \tau d s \\
& =1-\frac{2}{h_{n}} \int_{0}^{h_{n}} \int_{0}^{1} u \mu_{n}(d u, s) d s=1-2 \Theta_{n}\left(h_{n}\right) .
\end{aligned}
$$

Последняя формула влечет утверждение следствия 2.

Доказательство теоремы 3 проводится так же, как и доказательство теоремы 1 , только с использованием следующего представления:

$$
\begin{aligned}
S_{n}^{+} f(t)= & \sum_{k=0}^{m_{t}-1} f_{n}\left(Z_{n}\left(\tau_{t}+(k+p+2) h_{n}-, \tau_{t}+(k+1) h_{n}\right)\right) \\
& \times\left(Z_{n}\left(\tau_{t}+(k+p+2) h_{n}-, \tau_{t}+(k+1) h_{n}\right)\right. \\
& \left.\quad-Z_{n}\left(\tau_{t}+(k+p+2) h_{n}-, \tau_{t}+k h_{n}\right)\right) .
\end{aligned}
$$


Утверждение теоремы 4 вытекает из теоремы 3 в результате применения методов доказательства теоремы 2.

Следствия 3 и 4 доказываются аналогично предыдущим следствиям.

\section{СПИСОК ЛИТЕРАТУРЫ}

1. Itô K. Stochastic integral. - Proc. Imp. Acad. Tokyo, 1944, v. 20, p. 519-524.

2. Fisk D. L. Quasi-martingales and stochastic integrals. - Tech. Rep., v. 1. Dept. Math. Michigan State Univ., 1963.

3. Стратонович Р. Л. Новая форма записи стохастических интегралов и уравнений. - Вестник Моск. ун-та, 1964, т. 1, с. 3-12.

4. Ogawa S. On a Riemann definition of the stochastic integral. I, II. - Proc. Japan Acad., 1970, v. 46, № 21, p. 153-157; p. 158-161.

5. Пугачев В. С., Синицын И. Н. Стохастические дифференциальные системы. Анализ и фильтрация. М.: Наука, 1990, 630 с.

6. Гихман И.И., Скороход А.В. Стохастические дифференциальные уравнения и их приложения. Киев: Наукова думка, 1982, 611 с.

7. Wong E., Zakai M. On the convergence of ordinary integrals to stochastic integrals. Ann. Math. Statist., 1965, v. 36, № 5, p. 1560-1564.

8. Ватанабэ $C$., Икэда $H$. Стохастические дифференциальные уравнения и диффузионные процессы. М: Наука, 1986, 445 с.

9. Protter P. Stochastic Integration and Differential Equations: A New Approach. Berlin: Springer-Verlag, 1990, 302 c.

10. Лазакович Н. В. Стохастические дифференциалы в алгебре обобщенных случайных процессов. - Докл. АН Беларуси, 1994, т. 38, № 5, с. 23-27.

11. Лазакович Н.В., Сташуленок С. П., Стемковская Т. В. Ассоциированные решения уравнений в дифференциалах в прямом произведении алгебр обобщенных случайных процессов. - Теория вероятн. и ее примен., 1998, т. 43, в. 2, с. 272-293.

12. Владимиров В. С. Уравнения математической физики. М.: Наука, 1988, 512 с.

13. Лазакович Н. В., Сташуленок С. П., Яблонский О. Л. Некоторые аппроксимации стохастических $\theta$-интегралов. - Литов. матем. сб., 1999, т. 39, № 2, с. 248-256.

14. Яблонский О. Л. Классификация способов аппроксимации стохастических интегралов в алгебре обобщенных случайных процессов. - Докл. НАН Беларуси, 2000 , т. 44 , № 2, c. 23-25.

15. Лазакович Н. В., Яблонский О. Л. О приближении решений одного класса стохастических уравнений. - Сиб. матем. журн., 2001, т. 42, № 1, с. 87-102.

16. Lazakovich N. V., Yablonski A. L. On the approximation of the solutions of stochastic equations with $\theta$-integrals. - Stochastics Stochastics Rep., 2004, v. 76, № 2, p. 135145.

17. Лесневский В. Э. О приближении решений стохастических дифференциальных уравнений, содержаших случайный процесс Пуассона. - Докл. НАН Беларуси, 2000 , т. 44 , № 4, c. 34-36.

18. Marcus S.I. Modeling and approximation of stochastic differential equations driven by semimartingales. - Stochastics, 1981, v. 4, № 3, p. 223-245.

19. Kurtz T., Pardoux É., Protter P. Stratonovich stochastic differential equations driven by general semimartingales. - Ann. Inst. H. Poincaré, 1995, v. 31, № 2, p. 351-377.

20. Гихман И.И., Скороход А.В. Теория случайных процессов. Т. III. М.: Наука, $1975,496 \mathrm{c}$.

Поступила в редакцию 16.XII.2002

Исправленный вариант 10.V.2004 